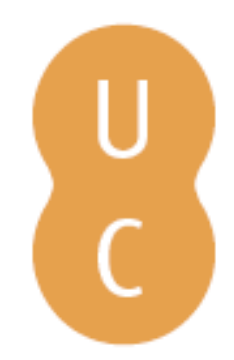

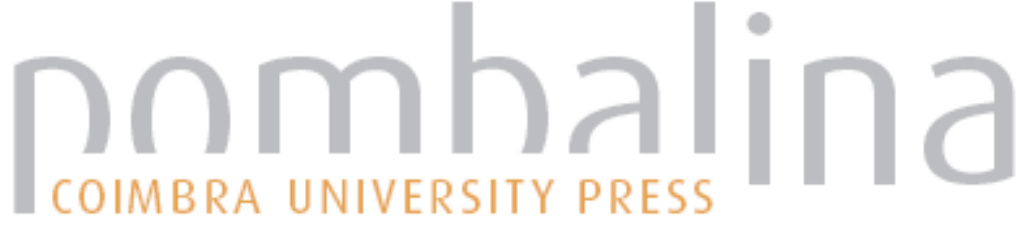

\section{Las rutas historiográficas de la farmacia en México Siglos XIX y XX}

Autor(es): $\quad$ Cosme, Alba Morales; Pastrana, Patricia Aceves

Publicado por: Imprensa da Universidade de Coimbra

URL persistente:

URI:http://hdl.handle.net/10316.2/32383

DOI:

DOl:http://dx.doi.org/10.14195/978-989-26-0469-5_9

Accessed : $\quad$ 26-Apr-2023 10:33:56

A navegação consulta e descarregamento dos títulos inseridos nas Bibliotecas Digitais UC Digitalis, UC Pombalina e UC Impactum, pressupõem a aceitação plena e sem reservas dos Termos e Condições de Uso destas Bibliotecas Digitais, disponíveis em https://digitalis.uc.pt/pt-pt/termos.

Conforme exposto nos referidos Termos e Condições de Uso, o descarregamento de títulos de acesso restrito requer uma licença válida de autorização devendo o utilizador aceder ao(s) documento(s) a partir de um endereço de IP da instituição detentora da supramencionada licença.

Ao utilizador é apenas permitido o descarregamento para uso pessoal, pelo que o emprego do(s) título(s) descarregado(s) para outro fim, designadamente comercial, carece de autorização do respetivo autor ou editor da obra.

Na medida em que todas as obras da UC Digitalis se encontram protegidas pelo Código do Direito de Autor e Direitos Conexos e demais legislação aplicável, toda a cópia, parcial ou total, deste documento, nos casos em que é legalmente admitida, deverá conter ou fazer-se acompanhar por este aviso. 
Ana Leunar Pereira João Rui Pita

[ Coordenaçä̃ ]
Rotas da Natureza

Cientistas

Viagens

Expedifgũes

Instituip̧ües

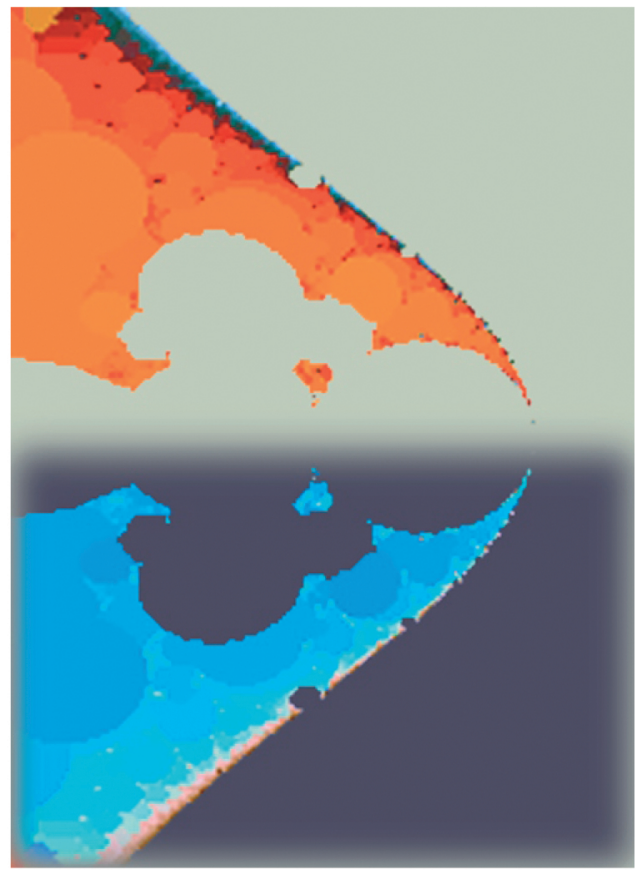




\section{Coordenaçáo Científica da Colecção Ciências e Culturas}

João Rui Pita e Ana Leonor Pereira

Os originais enviados são sujeitos a apreciação científica por referees

\section{Coordenação Editorial}

Maria João Padez Ferreira de Castro

\section{Edição}

Imprensa da Universidade de Coimbra

Email:impresauc@ci.uc.pt

URL: http://www.imp.uc.pt • Normas de publicação de colecçôes

\section{Design}

António Barros

Pré-Impressáo

António Resende

Imprensa da Universidade de Coimbra

\section{Capa}

António Barros, com imagem de E. M. de Melo e Castro, 2003 [Fractal original gerado no Fractint com tratamento no Photoshop 7.0]; Cortesia: António Barros

Impressão e Acabamento

SerSilito • Maia

\section{ISBN}

978-989-8074-12-6

\section{Depósito Legal}

Obra publicada com a colaboraçáo de:
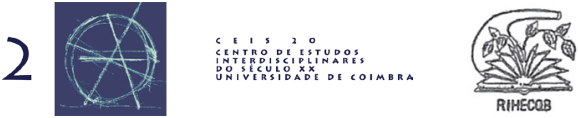

Obra publicada com o apoio de:

FCT Fundação para a Ciência e a Tecnologia

MINISTÉRIO DA CIÊNCIA, TECNOLOGIA E ENSINO SUPERTOR Portug

Programa Operacional Ciência, Tecnologia, Inovação do Quadro Comunitário de Apoio III
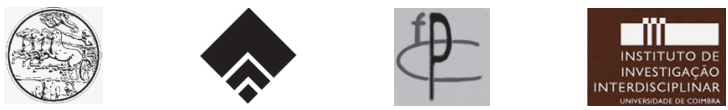

\section{Baxter}

(c) 2006, Imprensa da Universidade de Coimbra 
João Rui Pita

Ana Leonar Pereira

(Courdenação)

Rotas da Natureza

Cientistas

Viagens

Expediçũes

Instituiç̃̃es

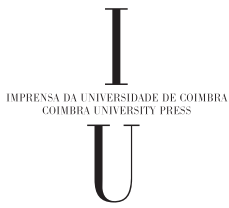

- colmbra 2006 
(Página deixada propositadamente em branco) 


\section{LAS RUTAS HISTORIOGRÁFICAS DE LA FARMACIA EN MÉXICO SIGLOS XIX Y XX}

La carrera de Farmacia se creó en 1833 en el Establecimiento de Ciencias Médicas. A principios del siguiente siglo, en 1919, los farmacéuticos abandonaron la Escuela de Medicina para unirse a la recién creada Facultad de Química, lo cual marcó una nueva etapa en la historia de su disciplina. El proceso por el que atraviesa la Farmacia en este periodo ha sido objeto de diversas investigaciones que han alimentado una novedosa línea en la historiografía mexicana de las ciencias; cuyo campo de estudio en construcción, ha ido conformando un acervo que da cuenta del desarrollo de la Farmacia desde finales del siglo XVIII hasta los principios del XX. La descripción - a grandes pinceladas - de las diversas etapas y áreas de interés involucradas en el pasado de esta disciplina, es el objetivo del presente trabajo.

\section{La farmacia en la historia: las primeras rutas}

El siglo XIX representa una etapa importante para la historia de la ciencia en México dado que durante la centuria se crearon nuevas formas de organización profesional, académica y legal que configuraron el ejercicio de la ciencia durante la etapa independiente $^{1}$. Así por ejemplo, se instituyeron sociedades especializadas, se multiplicaron las publicaciones, se conformaron las primeras instituciones de investigación y los hombres de ciencia se convirtieron en profesionales. En otras palabras, la ciencia se establece como institución dentro de la estructura social ${ }^{2}$.

En este marco, los profesionales de la farmacia constituían un minoría dentro de la reducida comunidad científica mexicana del siglo XIX, sus lazos de unión eran

\footnotetext{
${ }^{1}$ Azuela, Luz Fernanda; Guevara,Rafael, «La ciencia en México en el siglo XIX: una aproximación historiográfica", Asclepio, v. L, fasc. 2, 1998.

${ }^{2}$ La ciencia - dice Leonel Rodríguez - fue un elemento constitutivo en la concepción moderna del Estado. Ver: Rodríguez, Leonel, La ciencia y la técnica en la industrialización de México independiente: estudio histórico del programa editorial promovido por el Banco de Avio. 1830-1832, Tesis de Maestría en Ciencias, Instituto Politécnico Nacional, 2000, p. 7.
} 
precarios y el aprecio social hacia ellos era escaso. El anterior señalamiento podría explicar el por qué hasta hace poco la historia de la farmacia en el siglo XIX debía ser rastreada en las historias generales de la ciencia en México donde aparece ligada a la vida de personajes señeros como Leopoldo Río de la Loza (1807-1876), considerado el padre de la química, y Alfonso Herrera (1838-1931) fundador de la Sociedad de Historia Natural e impulsor del primer instituto de investigación en México, el Instituto Médico $\mathrm{Nacional}^{3}$. O bien, se le vincula a la práctica de disciplinas importantes en esta época como la medicina, la historia natural y la botánica. No es extraño que en esta línea historiográfica el desarrollo histórico de la Farmacia en México aparezca unido primero a la medicina y, a partir de la segunda década del siglo XX a la química; asimismo, en las historias generales de las ciencias, la farmacia también va relacionada con estas dos ciencias ${ }^{4}$.

Los trabajos realizados en México a partir de 1970, se dieron a la tarea de recuperar la producción científica local - es decir - las obras, publicaciones periódicas, y hombres de ciencia, cuya existencia cobraba significado en el propio contexto de estudio. Fue una primera forma en la que se intentó superar el enfoque eurocentrista, que en términos historiográficos implicaba buscar en nuestra historia rastros de la ciencia europea. El propósito era explicar la tradición científica nacional y en ese sentido, la historia social de la ciencia ha ofrecido una nueva línea para estudiar el ejercicio científico como una actividad señalada por el contexto en el que tiene lugar. Esta ruta desplaza el enfoque difusionista de la historia de la ciencia y deja de explicar la actividad científica del siglo XVIII y XIX como resultado de la inercia generada durante la Ilustración ${ }^{5}$. Para el caso de la Farmacia, la historia social ha permitido subrayar la importancia de la estructura institucional y de las formas de organización académicas y profesionales en donde aterriza la práctica científica.

\section{Las transiciones: del XVIII al XIX y del XIX al XX}

Los trabajos que han estudiado la farmacia entre los años que nos ocupan han privilegiado dos periodos históricos: el que explica el surgimiento de la carrera de farmacia y que parte de la segunda mitad del siglo XVIII; y aquellos que se ubican en el último tercio del siglo XIX, que en el contexto político corresponden a la República Restaurada (1867-1876) y al gobierno de Porfirio Díaz (1876-1910). Estas últimas son etapas en las que la estabilidad política permitió la construcción de programas científicos dentro de los cuales se ubica la Farmacia.

\footnotetext{
${ }^{3}$ De Gortari, Eli, La ciencia en la historia de México, México, Grijalbo, 1980.

${ }^{4}$ Sólo existe un estudio general dedicado a la historia de la farmacia en México realizado en 1992 por Pérez Islas, Valentín y Sánchez Ruiz, Juan F. Se trata de un texto revisionista que no aborda problemáticas específicas de la disciplina: Breve historia de la Farmacia en México y en el mundo, México, Asociación Farmacéutica Mexicana, 1992.

${ }^{5}$ Lafuente, A. «Ciencia colonial y roles socioprofesionales en la América Española del siglo XVIII», en Quipu, 6 (3) 1989; Chambers, W., "Localty and science: of center and periphery", Mundialización de la ciencia y cultura nacional, Madrid, Doce Calles, 1993, pp. 605-618.
} 
La ruta historiográfica que aborda el primer periodo ha generado trabajos que ilustran la noción de proceso que condujo a la creación de la carrera de Farmacia. En los textos se aborda la renovación de la práctica farmacéutica originada a raíz, por un lado, de la incorporación de nuevos saberes relacionados con la química y la botánica; y por otro, de la fundación de nuevas instituciones como el Hospital General de San Andrés (1779), el Jardín Botánico (1788), y su Cátedra de Botánica (1788), cuya aparición significó la alteración y progresivo rompimiento de la organización gremial de la disciplina ${ }^{6}$. Los estudios subrayan el papel destacado de la Farmacia en el cambio del modelo de organización profesional y administrativo de las disciplinas de la salud en la primera mitad del siglo XIX y enfatizan la importancia de los materiales culturales preexistentes y de formas de organización académico-profesionales locales ${ }^{7}$.

Dentro de la producción historiográfica de este periodo destaca un tema: la conformación de una materia médica nacional, tarea que ocupó a personajes como Vicente Cervantes, Antonio de la Cal y Leopoldo Río de la Loza, y dio pie al surgimiento de la primera Academia de Farmacia, la cual editó la primera Farmacopea Mexicana en $1846^{8}$. Este proyecto, significó rescatar los recursos terapéuticos locales, uniformar y sistematizar la práctica profesional, unificar el sector en la primera etapa independiente y, sobre todo, ofrecer la imagen de la Farmacia como ciencia nacional ${ }^{9}$.

Al lado de estos aspecto se han estudiado las reformas institucionales, la adopción de un nuevo lenguaje farmacéutico derivado de la introducción de la nomenclatura de Lavoisier, así como la denominada internacionalización de la ciencia, o bien los intercambios científicos manifestados en la presencia de una variada lista de libros de procedencia diversa, revistas y profesores extranjeros ${ }^{10}$.

\footnotetext{
${ }^{6}$ Se pueden citar los trabajos de: Aceves, Patricia, Química, botánica y farmacia en la Nueva España a finales del siglo XVIII, México, Universidad Autónoma Metropolitana, 1993; Huerta, Ana María, Los boticarios poblanos 1536-1825, Puebla, Gob. Del Edo de Puebla-Sria de Cultura, 1994. Azuela Luz Fernanda; Guevara,Rafael, «La ciencia en México en el siglo XIX: una aproximación historiográfica», Aclepio, v.L, fasc. 2, 1998,.

${ }^{7}$ Una obra muy completa que desarrolla estos aspectos es: Morales, Alba, El Hospital General de San Andrés: la modernización de la medicina novohispana (1770-1833), P. Aceves (ed.), Biblioteca Historia de la Farmacia, No. 2, México, UAM-Xochimilco, Soc. Quim. Mex., Colegio Nac. QFB., 2002.

${ }^{8}$ Ver: Urbán, Guadalupe, La obra cientifica el doctor Leopoldo Río de la Loza, Aceves, P. (ed y coord.), Biblioteca de Historia de la Farmacia, No. 1, México, UAM-Xochimilco, IPN, Soc. Quim. Mex., 2000.

${ }^{9}$ Aceves, Patricia. "Hacia una farmacia nacional: la primera farmacopea del México Independiente», en P. Aceves (ed.), Farmacia, historia natural y química intercontinentales, Estudios de Historia Social de las Ciencias Químicas y Biológicas, N. 3, México, UAM-Xochimilco, 1995, pp.161-178; Ana María Huerta ha contribuido con diversos trabajos de carácter regional muy valiosos por la carencia de este tipo de estudios en la historiografía del tema., entre ellos se encuentran: «La farmacia y las ciencias médicas en Puebla. 1795-1848», en P. Aceves (ed) Farmacia, historia natural y quimica intercontinentales, op. cit., pp. 179-188; "La primera materia médica del México independiente. Influencias y procedencias», en P. Aceves (ed), Tradiciones e intercambios científicos. Materia médica, farmacia y medicina, Estudios de Historia Social de las Ciencias Químicas y Biológicas, No 5, México, UAM-Xochimilco, Instituto Politécnico Nacional, Sociedad Química de México, A.C., 2000, pp. 301-316; El Jardín de la Cal. Antonio de la Cal y Bracho, la botánica y las ciencias de la salud en Puebla, Puebla, Gob. Del Edo de Puebla-Sria. de Cultura, 1996.

${ }^{10}$ Lavoisier A. L., Tratado elemental de chimica, México, Mariano Zúñiga y Ontiveros, 1797, (edición facsimilar con un Estudio preliminar de P. Aceves P., México, UAM-Xochimilco, 1990); Empotz, Gerard y Aceves, P. (eds), Between the Natural and Artificial Dyestuffs and Medicines, Collection de Travaux de l'Academie
} 
Otro periodo bien estudiado desde la historia de la ciencia en general y de la farmacia en particular, es el correspondiente al último tercio del siglo XIX y las primeras décadas del XX. Para abordarlo se parte del argumento de que la estabilidad política permitió al Estado configurar políticas científicas, en cuya construcción participaron los propios hombres de ciencia. En ese sentido se han analizado las relaciones de los farmacéuticos con el poder político en estos años ${ }^{11}$, aspecto desde el cual se explica la consolidación del proceso de institucionalización de la disciplina; hecho que se manifiesta en la conformación de la Sociedad Farmacéutica Mexicana (1871) y la aparición del primer órgano de difusión de la disciplina: la revista La Farmacia, publicada a partir de 1891.

En este periodo, además de las figuras señeras de la Farmacia, como Alfonso Herrera, o bien las propias instituciones que abren espacios para la práctica farmacéutica (la Sociedad, la revista La Farmacia, las cátedras de la Escuela de Medicina) destaca como sujeto de la historia el Estado; entidad que tiene un papel central en el caso de la ciencia en México ${ }^{12}$.

Diversos trabajos monográficos tratan los aspectos sobresalientes en el camino recorrido por la Farmacia para su profesionalización ${ }^{13}$, y han abierto otras vertientes que analizan la estructura de la enseñanza y las tesis de grado (requisito impuesto a los farmacéuticos en 1870) ${ }^{14}$. Por último, recientemente ha sido abordada la construcción de identidades profesionales, ruta que pasa por la revisión de los saberes propios de la Farmacia, sus campos de trabajo y los escenarios institucionales para su ejercicio. Esta orientación ha sido tomada para explicar, por ejemplo, el viraje dado por la Farmacia mexicana al pasar del ámbito médico al químico a partir del último tercio del siglo XIX ${ }^{15}$.

Internationale d'Histoire des Sciences, Turnhout; Brepols Publishers, 2000; Aceves, P. «Bibliografía médicofarmacéutica del siglo XVIII novohispano», en P. Aceves (ed), Construyendo las ciencias químicas y biológicas, Estudios de Historia Social de las Ciencias Quimicas y Biológicas, No. 4, México, UAM-X., 1998, pp. 99-120; Saladino, Alberto, "Bibliografía farmacéutica durante el siglo XVIII en la América Hispánica», en Ibid, pp. $121-138$.

${ }^{11}$ Azuela, L. F. y R. Guevara «Las relaciones entre la comunidad científica y el poder político en México en el siglo XIX, a través del estudio de los farmacéuticos», en P. Aceves (ed), Construyendo las ciencias químicas y biológicas, Estudios de Historia Social de las Ciencias Químicas y Biológicas, op. cit, pp. 239258.

${ }^{12}$ Ver: Aceves, P. y Olea, Adolfo (coords.), Alfonso Herrera: homenaje a 100 años de su muerte, Biblioteca Historia de la Farmacia, No. 5, México, UAM-Xochimilco, Soc. Quim. Mex., Colegio Nac. QFB., 2002.

${ }^{13}$ Hinke, Nina "Entre arte y ciencia: la farmacia en México a finales del siglo XIX», en Relaciones. Estudios de Historia y sociedad. Ciencia y Nación en México, El Colegio de Michoacán, v. 22, n. 88, (oct-dic) 2001, pp. 51-78.

${ }^{14}$ Ortiz, Mariana, Las tesis de farmacia del siglo XIX mexicano, Aceves P. (ed.), Biblioteca de Historia de la Farmacia, No.4, México, UAM-Xochimilco, Soc. Quim. Mex., Colegio Nac. QFB, 2002.

${ }_{15}$ Martínez, Sandra, Desarrollo y transformación de la farmacia en México (1890-1920). El caso de las primeras mujeres farmacéuticas. Tesis de Licenciatura en Historia, México, Facultad de Filosofía y Letras, UNAM, 2003. 


\section{Comentario final}

Las líneas de estudio reseñadas a grandes rasgos, ilustran el camino seguido por una disciplina poco estudiada en la historia de la ciencia en México: la Farmacia. La construcción y reconstrucción de este objeto de estudio ha redundado no sólo en la renovación de los sujetos de la historia de la ciencia en México, sino también en la puesta al día de las fuentes de estudio, los personajes, las instituciones y los enfoques.

Por su condición de reciente, la historiografía de la Farmacia escapó a los debates de internalistas y externalistas y a los enfoques positivistas y difusionistas. Adscrita a la llamada historia social de la ciencia, ha ofrecido una nueva perspectiva para señalar la importancia de la estructura institucional y de las formas de organización académicas y profesionales de la práctica farmacéutica ${ }^{16}$. De esta manera los trabajos de historia de la Farmacia se han tornado cada vez más específicos y se han ocupado en definir los procesos de enseñanza, profesionalización, institucionalización y organización de redes científicas; así como la conformación de identidades profesionales. Además, la existencia de estudios regionales al respecto permite un acercamiento mayor y más real del hecho histórico, y proporciona un plano de reflexión diferente que enriquece la historia de la ciencia en México.

${ }^{16}$ Muchos de los trabajos que abren nuevas rutas para la historia de la ciencia han tenido que construir espacios para su exposición, este es el caso de las colecciones: Estudios de Historia Social de las Ciencias Químicas y Biológicas que integra 7 volúmenes y de Biblioteca de Historia de la Farmacia que cuenta con 5 volúmenes. 


\section{Colecçãa \\ 2 Ciências e Culturas Caimbra 2006}

